\title{
DNA damage and S phase-dependent E2F1 stabilization requires the cIAP1 E3-ubiquitin ligase and is associated with K63-poly-ubiquitination on lysine $161 / 164$ residues
}

\author{
Valérie Glorian ${ }^{1,2}$, Jennifer Allègre ${ }^{1,2}$, Jean Berthelet ${ }^{1,2}$, Baptiste Dumetier ${ }^{1,2}$, Pierre-Marie Boutanquoi ${ }^{1,2}$, Nathalie Droin ${ }^{3}$, Cémile Kayaci ${ }^{1,2}$, \\ Jessy Cartier ${ }^{1,2}$, Simon Gemble ${ }^{1,2}$, Guillaume Marcion ${ }^{1,2}$, Daniel Gonzalez ${ }^{1,2,4}$, Romain Boidot ${ }^{1,2,4}$, Carmen Garrido ${ }^{1,2}$, Olivier Michaud ${ }^{1,2}$, \\ Eric Solary ${ }^{3,5}$ and Laurence Dubrez ${ }^{*, 1,2}$
}

The E2F transcription factor 1 is subtly regulated along the cell cycle progression and in response to DNA damage by posttranslational modifications. Here, we demonstrated that the E3-ubiquitin ligase cellular inhibitor of apoptosis 1 (cIAP1) increases E2F1 K63-poly-ubiquitination on the lysine residue 161/164 cluster, which is associated with the transcriptional factor stability and activity. Mutation of these lysine residues completely abrogates the binding of E2F1 to CCNE, TP73 and APAF1 promoters, thus inhibiting transcriptional activation of these genes and E2F1-mediated cell proliferation control. Importantly, E2F1 stabilization in response to etoposide-induced DNA damage or during the $S$ phase of cell cycle, as revealed by cyclin $A$ silencing, is associated with K63-poly-ubiquitinylation of E2F1 on lysine 161/164 residues and involves cIAP1. Our results reveal an additional level of regulation of the stability and the activity of E2F1 by a non-degradative K63-poly-ubiquitination and uncover a novel function for the E3-ubiquitin ligase cIAP1.

Cell Death and Disease (2017) 8, e2816; doi:10.1038/cddis.2017.222; published online 25 May 2017

The E2 factor (E2F) protein family consists of eight transcription factors (E2F1-8) that are essential regulators of cell proliferation, cell differentiation, DNA damage response, cell death and cell senescence. Their foremost functions are in cell cycle regulation as they participate in the dynamic expression of molecules driving cell cycle progression. E2F1 is the most extensively studied member of this family. Its protein expression level is increased in many human cancers such as hepatocarcinomas, digestive adenocarcinomas and ovarian cancers, and its downregulation in p53- and RB-compromised cancer cells can trigger their senescence. ${ }^{1}$ Further understanding of E2F1 expression regulation would help to develop strategies targeting this protein in cancer cells.

The expression and activity of E2F1 are regulated through post-translational modifications and association with different regulatory factors. E2F1 transcriptional activity is repressed by binding to the hypo-phosphorylated form of the pocket protein $\mathrm{pRb}$ (retinoblastoma protein). ${ }^{2}$ In the late $\mathrm{G} 1$ phase of cell cycle, the phosphorylation of $\mathrm{Rb}$ by cyclin/cdk complexes releases $\mathrm{E} 2 \mathrm{~F} 1$, allowing the transcription transcription of genes required for $\mathrm{G} 1-\mathrm{S}$ phase transition and DNA replication. ${ }^{2}$ E2F1 is subsequently degraded in late $S$ and G2 phases by the ubiquitin proteasome system (UPS). ${ }^{3} \mathrm{~A}$ genotoxic stress can also induce the stabilization and activation of E2F1 that, in this situation, promotes cell death rather than cell proliferation. ${ }^{4}$ The turnover and activity of E2F1 are regulated by a number of post-translational modifications including phosphorylation, acetylation, methylation, neddylation, sumoylation and ubiquitination. ${ }^{2,4}$

Ubiquitination consists of a multistep enzymatic process, the aim of which is to conjugate ubiquitin molecules on a lysine of target proteins. This process is mediated by a cascade reaction involving an ubiquitin-activating enzyme (E1), an ubiquitin-conjugating enzyme (E2) and an E3-ubiquitin ligase enzyme or E3-ubiquitin ligase enzymatic complex. The free $\mathrm{N}$-terminus of ubiquitin or each of its seven lysine residues can act as an acceptor site for ubiquitin. At least eight types of ubiquitin chains can be formed depending on the ubiquitin acceptor residue. The nature of the ubiquitin chain defines the functional consequences of target protein ubiquitination. For example, Lys48 (K48) or Lys11 (K11)-linked chains promote the protein degradation by the proteasome, whereas Lys63 (K63)-assembled chains favor the protein recruitment into signaling platforms. ${ }^{5}$ E2F1 is ubiquitinated by the SCF ${ }^{\text {skp2 }}$ (Skp2 (S phase kinase binding protein 2)-CDC53 (Cullin)-Fbox) E3 ligase complex in the late $S$ and $G 2$ phase of cell cycle $^{6}$ and by APC/C ${ }^{\text {cdc20 }}$ (anaphase-promoting complex/ cyclosome) in early mitosis. ${ }^{7}$ In addition, in early $\mathrm{G} 1$, the $\mathrm{APC} / \mathrm{C}^{\mathrm{cdh} 1} \mathrm{E} 3$ ligase complex promotes the conjugation of the $\mathrm{K} 11$-linked ubiquitin chain on $\mathrm{E} 2 \mathrm{~F} 1 .^{8}$ In all these situations, ubiquitinated E2F1 is delivered to proteasome for degradation. A K63 ubiquitination of E2F1 has also been reported ${ }^{9,10}$ but

\footnotetext{
${ }^{1}$ Université de Bourgogne Franche-Comté, LNC UMR1231, Dijon, France; ${ }^{2}$ Institut National de la Santé et de la Recherche Médicale (Inserm), LNC UMR1231, Dijon, France; ${ }^{3}$ Inserm U1170, Gustave Roussy, Villejuif, France; ${ }^{4}$ Centre Georges-François Leclerc, Dijon, France and ${ }^{5}$ Université Paris-Sud, Faculté de Médecine, Le Kremlin Bicêtre, France

${ }^{*}$ Corresponding author: L Dubrez, Inserm LNC UMR1231, Faculty of Medicine, 7 Boulevard Jeanne d'Arc, Dijon Cedex 21079, France. Tel: +33 380393356 ; Fax: +33 380 393 434; E-mail: Idubrez@u-bourgogne.fr

Received 16.3.17; revised 06.4.17; accepted 13.4.17; Edited by G Raschellà
} 
the involved E3-ubiquitine ligase enzyme and the consequences of this modification remain unexplored.

Cellular inhibitor of apoptosis 1 (clAP1) is a RINGcontaining E3-ubiquitin ligase of the IAP family. Thanks to its ability to induce the ubiquitination of signaling molecules, clAP1 is a very potent regulator of the tumor necrosis factor receptor (TNFR) superfamily and NF- $K B$ transcription factoractivating signaling pathways. ${ }^{11}$ Therefore, clAP1 is an important regulator of immune and inflammatory responses. In undifferentiated healthy cells and in some tumor cells, clAP1 is expressed in the cell nucleus ${ }^{12-15}$ where it interacts with E2F1 protein. ${ }^{12}$ In this study, we demonstrate that clAP1 induces an accumulation of E2F1 modified through K63 ubiquitination on the lysine cluster $161 / 164$, which is preceded by an arginine methylation step. The mutation of both lysine residues completely abolishes the transcriptional activity of E2F1 and its recruitment onto target gene promoters. A clAP1-dependent accumulation of K63-ubiquitinated E2F1 is observed in the S phase of the cell cycle, when E2F1 activity is maximal and is required for the stabilization and the activation of E2F1 upon genotoxic stress.

\section{Results}

cIAP1 increases E2F1 protein expression and ubiquitination in an E3 ligase-dependent manner. We previously demonstrated that clAP1 is able to interact with E2F1 transcription factor and to increase its transcriptional activity. $^{12}$ Given that clAP1 is an E3-ubiquitin ligase, we assessed the role of ubiquitination reaction by using a pan-E1ubiquitin enzyme inhibitor PYR-41. PYR-41 repressed, in a dose-dependent manner, clAP1-mediated E2F1 transcriptional activation as analyzed using a cycline $\mathrm{E}$ gene reporter assay (Figure 1a). This E2F1 transcriptional activation is associated with a drastic increase in E2F1 expression level (Figures 1a, b and d), as well as an increase in E2F1 polyubiquitination (Figure 1c) as determined by pulling-down ubiquitinated proteins using tandem ubiquitin-binding entities (TUBEs). As non-degradative ubiquitination likely involves K63-linkage poly-ubiquitin chain, we confirmed our results by pulling-down ubiquitinated proteins using K63-linkage-specific TUBEs (Figure 1c). Importantly, these properties are lost when the clAP1 F616A mutation prevents the formation of IAP dimers (Figures $1 b$ and $c$ ). Consistent with the necessity of cIAP1 dimerization for its E3-ubiquitine ligase activity, ${ }^{16}$ the expression of the E3-ubiquitin ligase-deficient cIAP1 (H588A) conserved its capacity to stimulate E2F1 transcriptional activity in clAP1-expressing HeLa cells (Figure 1b) but not in clAPdeficient murine embryonic fibroblasts (Figure 1d). This suggest that, in HeLa cells, the E3 ligase defective H588A CIAP1 is likely to dimerize with the endogenous clAP1 to enhance E2F1 protein level and to stimulate its activity in an E3 ligase-dependent manner. Interestingly, the expression of clAP1 F616A induces a weak decrease in E2F1 expression (Figures $1 b, c$ and e). Treatment of cells with the proteasome inhibitor MG132 stabilizes the expression of both E2F1 and clAP1 and completely masks the effect of wt and the F616A clAP1 on E2F1 expression (Figure 1e), suggesting a stabilization of E2F1 by clAP1 and a proteasome-mediated degradation of E2F1 in the presence of the clAP1-F616A mutant that could acts as a dominant-negative form. Finally, using an in vitro ubiquitin assay, we could demonstrate that E2F1 is indeed a substrate of the E3-ubiquitine ligase cIAP1 (Figure 1f).

The cIAP1-mediated activation of E2F1 is associated with K63-poly-ubiquitinating on clustered lysines 161 and 164. The E2F1 protein contains 14 lysine residues, which are all potential acceptors for ubiquitin chains (Figure 2a). We constructed E2F1 mutants in which each lysine residue was replaced by an arginine residue and analyzed the capacity of clAP1 to stimulate the transcriptional activity of these E2F1 mutants using a gene reporter assay (Figure $2 b$ ). Whereas the steady-state level of these mutants was different, no correlation was observed between the level of expression of E2F1 and its transcriptional activity (Figure 2b). As already reported, ${ }^{17}$ mutation of the lysine cluster $117 / 120 / 125$, which is an acetylation site, strongly increased E2F1 transactivation activity (Figure $2 \mathrm{~b}$ ). The ability of clAP1 to promote wt E2F1 transcriptional activity was retained by all E2F1 mutants, except the K161R/164R mutant for which clAP1 was completely inactive and the K185R whose basal transactivation activity was weakly enhanced by clAP1 (Figure 2b). An analysis of the ubiquitin profile of E2F1 in HeLa cells revealed that cIAP1 promoted the accumulation of the polyubiquitinated forms of E2F1 wt and K137R mutant, but it failed to increase the poly-ubiquitination profile of the K161R/ K164R E2F1 mutant (Figures 2c and d), although it can still bind the protein, as demonstrated by co-immunoprecipitation (Figure 2e). Curiously, the K161/164R E2F1 mutant was strongly expressed and ubiquitinated at the basal state (Figures 2c and d). Finally, clAP1 bound and promoted the accumulation of the ubiquitinated K185R E2F1 mutant as efficiently as the E2F1 wt (Supplementary Figure 1). Altogether, our results suggested that the clAP1-mediated E2F1 transcriptional activity required ubiquitination on clustered lysine residues 161/164, located within the DNA-binding domain.

The E2F1 lysine cluster $161 / 164$ is required for E2F1 transcriptional activity. Mutations of the lysine residues of the cluster 1 (cl1: K117/K120/K125), cluster 2 (cl2: K182/ K183/K185) and/or cluster 3 (cl3: K226/K287/K289/K310) (Figure 2a) conserve $70-85 \%$ of E2F1 transcriptional activity as compared with the wt protein (Figure 3a). Remarkably, additional mutations at residues $\mathrm{K} 161$ and K164 markedly disrupted the E2F1 transcriptional activity (Figure 3a). As observed above, the constructs that contain the $\mathrm{K} 161 \mathrm{R} /$ K164R mutation are strongly expressed as compared with their control counterparts (Figure 3a). Whereas E2F1 constructs that contain no (K0), only one (K89) or only 2 (K89 and K137) lysine residues display a very weak transcriptional activity, restoration of $\mathrm{K} 161$ and $\mathrm{K} 164$ was sufficient to restore up to $75 \%$ of the E2F1 transcriptional activity (Figure $3 b$ ), highlighting the importance of these two residues. To further study the role of these residues and because HeLa cells express E6 and E7 proteins from human papillomaviruses that could affect the activity of E2F1, we used U2OS cells for functional analysis. In line with our 
a

HeLa

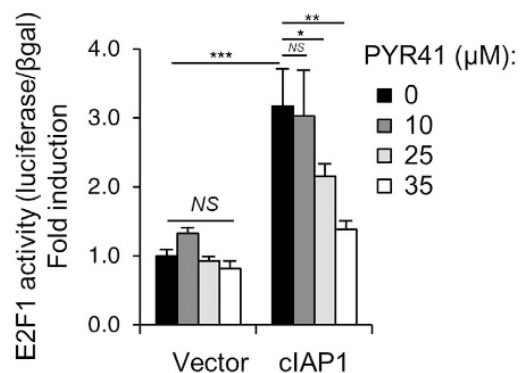

PYR41 ( $\mu \mathrm{M}): \begin{array}{lll}0 & 35 & 0 \quad 35\end{array}$

ClAP1 -70

E2F1 - - - 70 (3HA-E2F1)

$\beta$-actin

b

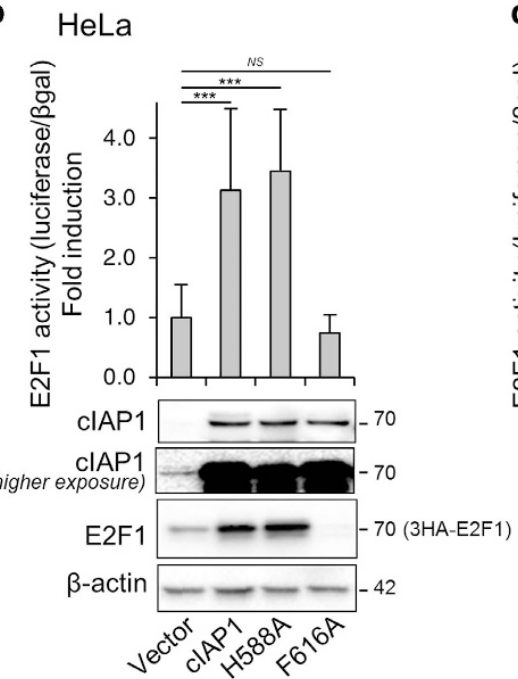

C

HeLa
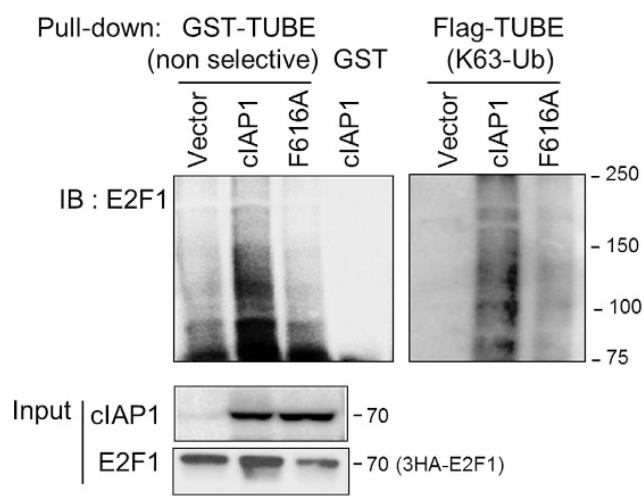

d KO MEFs

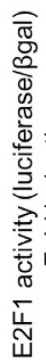

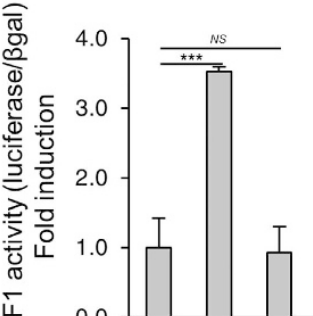

ClAP1 $\div-70$

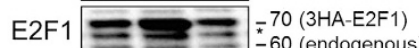

HSC70

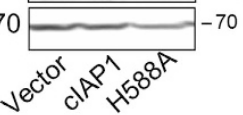

e HeLa

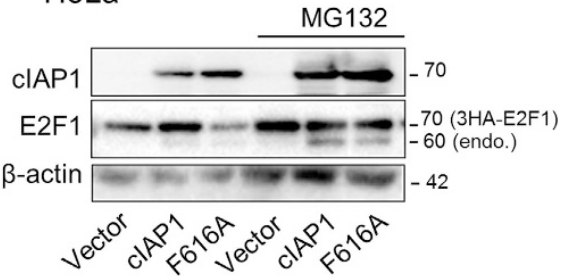

f

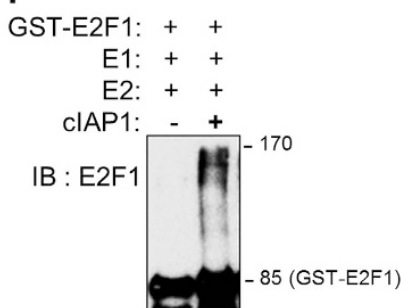

Figure 1 clAP1 stabilizes and stimulates E2F1 in an E3-ubiquitin ligase activity-dependent manner. (a, b and d) E2F1 activity and expression in Hela cells (a and $\mathbf{b})$ or in cIAP1 $1^{-1-} / \mathrm{ClAP2} 2^{-1-} \mathrm{MEFs}$ (d) transfected with CCNE promoter-Firefly luciferase reporter plasmid, pCMV-3HA-E2F1, along with empty vector (Vector), clAP1, CIAP1-H588A (devoid of E3-ubiquitin ligase activity) or cIAP1-F616A (lacking dimerization capacity) mutant encoding vector. HeLa cells were treated for $24 \mathrm{~h}$ with PYR-41 10-35 $\mu \mathrm{M}$ before analysis (a). Upper panels: E2F1 transcriptional activity was assessed in gene luciferase experiments. Luciferase activity was normalized to $\beta$-galactosidase activity and expressed as fold induction of promoter stimulated by E2F1 alone. Mean \pm S.D. of at least three independent experiments. Statistical analysis performed using Student's $t$-test. ${ }^{* * *} P<0.001,{ }^{* *} 0.001<P<0.01,{ }^{*} 0.01<P<0.1$, NS $P>0.1$. Lower panels: the expression of the constructs was analyzed by a western blot analysis. $\beta$-Actin or HSC70 are used as loading control. *Unspecific bands. (c) Ubiquitination assay performed in HeLa cells transfected with 3HA-E2F1, His-Ubiquitin encoding vectors, with an empty vector, CIAP1 or clAP1 F616A (F/A) mutant constructs. Ubiquitinated proteins were pulled-down by using non-selective or K63-TUBEs and ubiquitinated E2F1 is revealed by using an anti-E2F1 antibody. (e) Western blot analysis of clAP1 or 3HA-E2F1 expression in HeLa cells transfected with pCMV-3HA-E2F1 and with empty vector (Vector), clAP1 or CIAP1-F616A mutant (dimerization defective mutant) encoding vector, treated or not with MG132 $5 \mu \mathrm{M}$ overnight. (f) In vitro ubiquitination assay of GST-E2F1 fusion protein immobilized on gluthatione sepharose and incubated with ubiquitin, E1 and E2 recombinant proteins with or without recombinant clAP1

previous results (Figures $3 a$ and $b$ ), mutation of $\mathrm{K} 161$ and K164 to arginine was sufficient to completely abolish E2F1 transcriptional activity as monitored with CCNE, TP73 and APAF1 mRNA expression levels (Figure 3d). Moreover, the $\mathrm{K} 161 \mathrm{R} / \mathrm{K} 164 \mathrm{R}$ mutant was unable to bind the corresponding gene promoters (Figure $3 e$ ), although it was still found to be able to bind to DP1, a co-factor that is required for the binding of E2F1 to the DNA (Supplementary Figure 2). We next expressed E2F1 wild-type or the K161R/K164R double mutant in U2OS cells and measured cell proliferation using a crystal violet staining (Figure 3f). E2F1 overexpression was found to significantly slow down cell proliferation, whereas the mutant did not. Overall, our data suggest that K63 ubiquitination on cluster lysine residues 161/164 may be required for E2F1-mediated gene transcriptional regulation.

DNA damage induces clAP1-dependent accumulation of K63-ubiquitinated E2F1. We next evaluated the role of clAP1 and ubiquitination of E2F1 in DNA damage-induced stabilization of the protein, which is illustrated here in U2OS cells treated with increasing concentrations of the topoisomerase inhibitor etoposide (Figure 4). Depletion of clAP1 induced both a decrease in basal E2F1 expression levels and impaired etoposide-mediated E2F1 upregulation (Figure 4a). Etoposide treatment induced the accumulation of K63-ubiquitinated E2F1 proteins with an ubiquitination profile equivalent to the one 
a

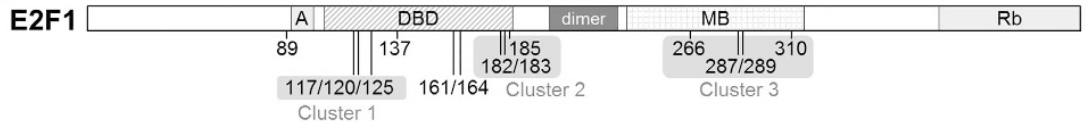

b

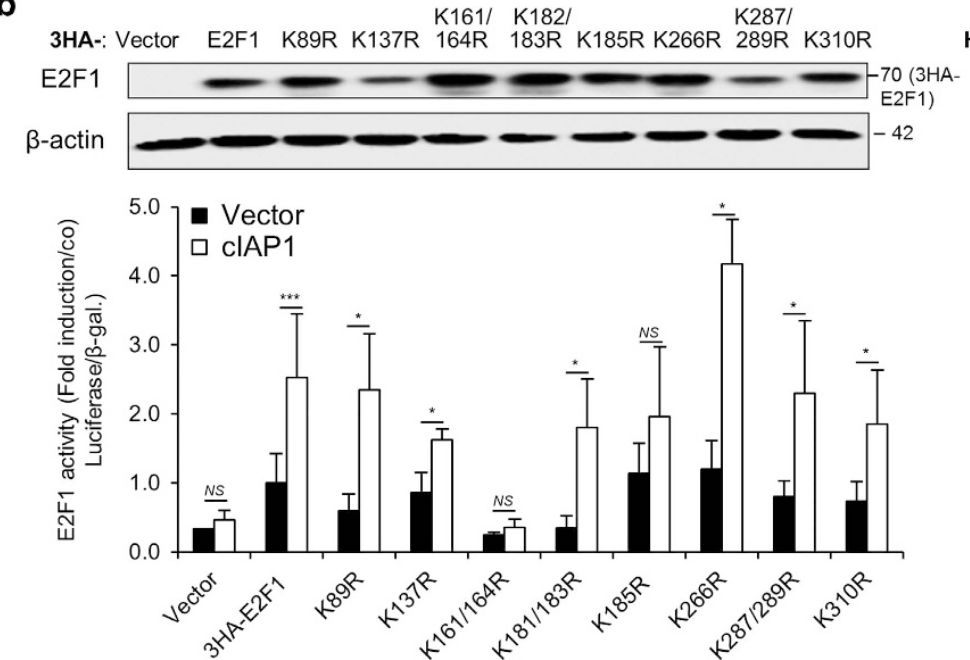

HA: Vector E2F1 $120 / 125 R$
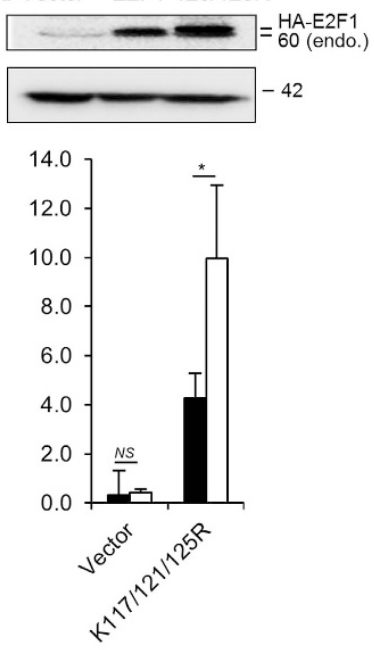

C

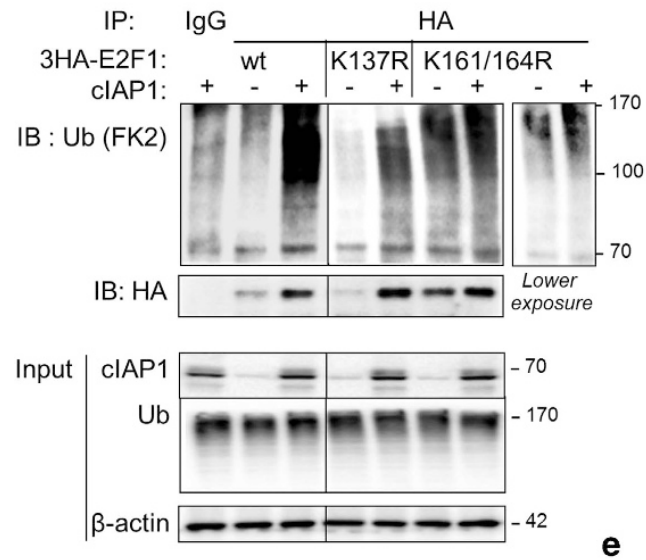

\section{d}
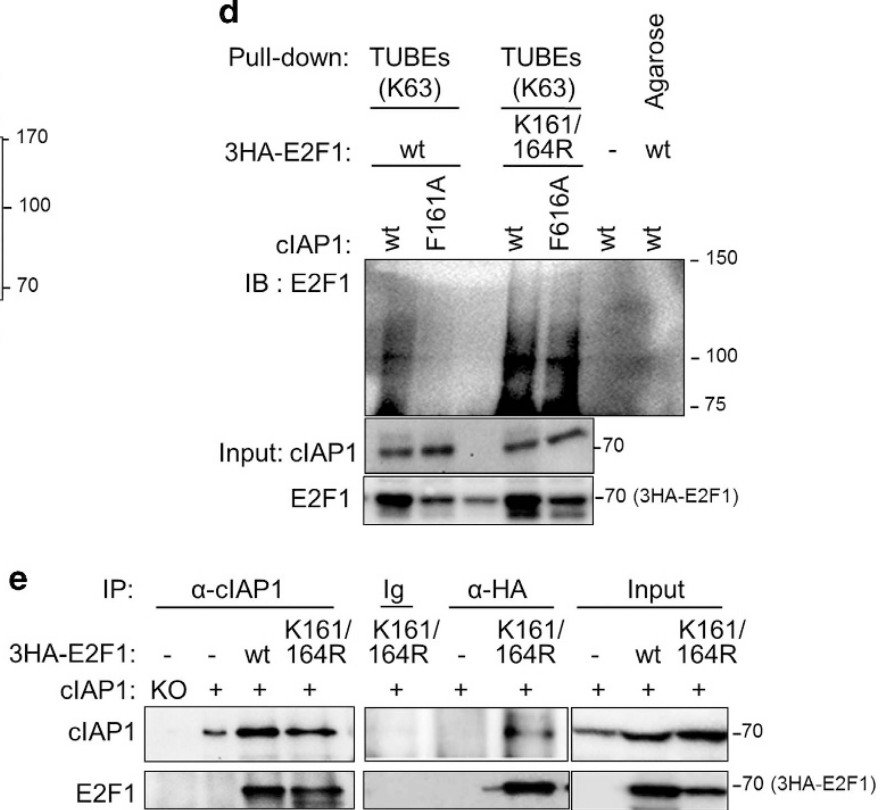

Figure 2 CIAP1 overexpression revealed a K63 ubiquitination of E2F1 on lysine residue 161/164. (a) Schematic representation of E2F1 protein structure indicating the lysine residues and lysine clusters. (a) Cyclin A: CDK2-binding domain; DBD: DNA-binding domain, dimer: dimerization domain; MB: marked box: Rb: Rb-binding domain. (b) Gene luciferase experiments performed in HeLa cells transfected with CCNE promoter-Firefly luciferase reporter plasmid, pCMV-HA (vector), HA-tagged-E2F1 (one single or 3HA) or HA-E2F1 mutants in which indicated $\mathrm{K}$ have been mutated into R along with empty vector (vector) or cIAP1-encoding vector. Luciferase activity was normalized to $\beta$-galactosidase activity and expressed as fold induction of promoter stimulated by E2F1. Mean \pm S.D. of at least three independent experiments. Statistical analysis performed using Student's $t$ test. ${ }^{* \star \star} P<0.001$, ${ }^{*} 0.01<P<0.1$, NS $P>0.1$. The expression of the E2F1 constructs was checked by a western blot analysis (upper panel). $\beta$-Actin is used as loading control. (c and d) Ubiquitination assay performed in HeLa cells transfected with 3HA-E2F1 wt, K137R mutant or K161/164R mutant, His-Ubiquitin, with an empty vector or ClAP1 or clAP1 F616A (F/A) mutant constructs. E2F1 is IP by using specific anti-E2F1 antibody and ubiquitination is revealed by using pan-ubiquitin antibody (FK2) (c), or ubiquitinated proteins were pulled-down by using K63-specific TUBEs and ubiquitinated E2F1 is revealed by using an anti-E2F1 antibody (d). (e) IP analysis of the interaction of CIAP1 with E2F1 wt or K161/164 R mutant. Hela cells were transfected with 3HA-E2F1 encoding constructs and CIAP1. cIAP1 or E2F1 were IP using anti-cIAP1 or anti-HA antibody and clAP1-E2F1 interaction was revealed by a western blot analysis. KO: cell lysate from clAP1 ${ }^{-1}-$ KO MEFs was used to check nonspecific reactivity of the antibody 
observed after clAP1 overexpression, an effect prevented by clAP1 silencing (Figures $4 \mathrm{~b}$ and $\mathrm{c}$ ). Contrary to wt E2F1, the $\mathrm{K} 161 \mathrm{R} / \mathrm{K} 164 \mathrm{R}$ double mutant failed to undergo stabilization
(Figure 4d) and K63 ubiquitination (Figure 4e) in response to etoposide treatment. Consistently, depletion of clAP1 by using either a siRNA or GDC-0152, an IAP antagonist known to a

HeLa
3HA- or Flag-E2F1: wt

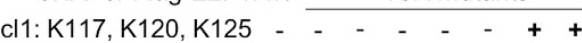
Cl2: K182, K183, K185 - $\quad-\quad+\quad+\quad+$ $\mathrm{cl3:} \mathrm{K} 226, \mathrm{~K} 287, \mathrm{~K} 289, \mathrm{~K} 310,-++++++$
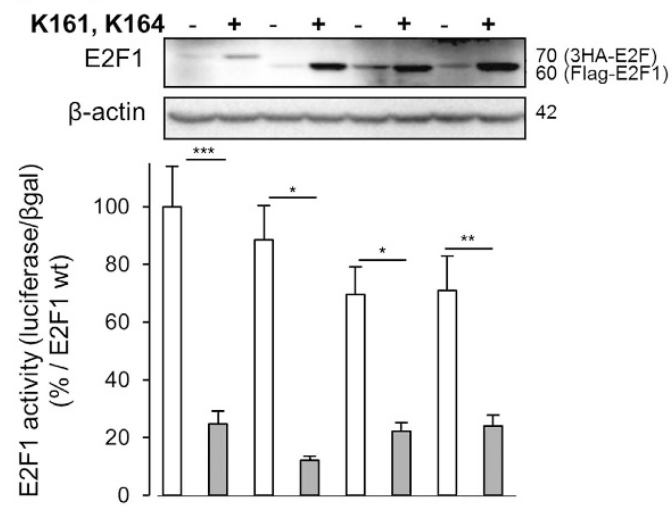

b

HeLa

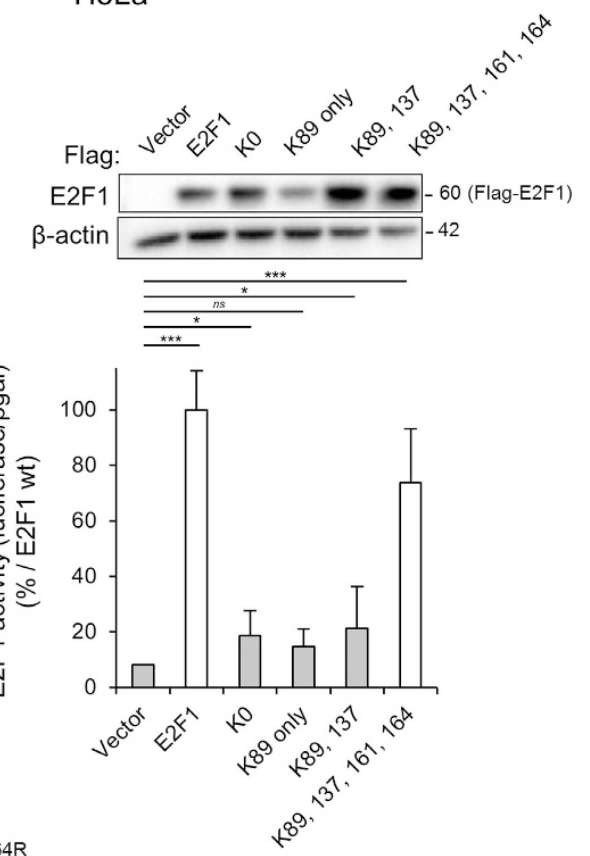

C

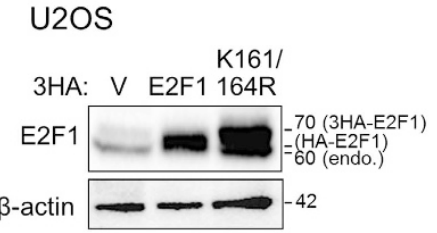

e U2OS

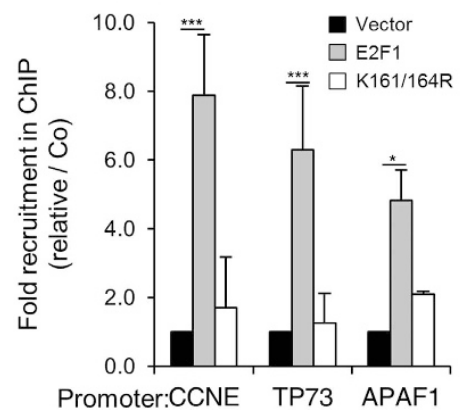

d U2OS

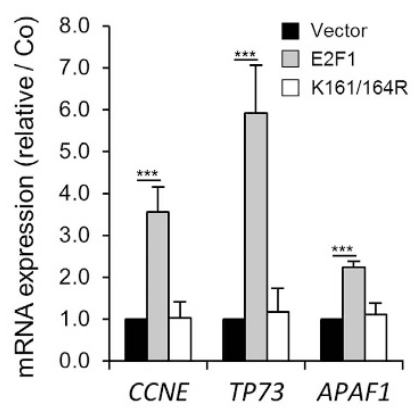

f

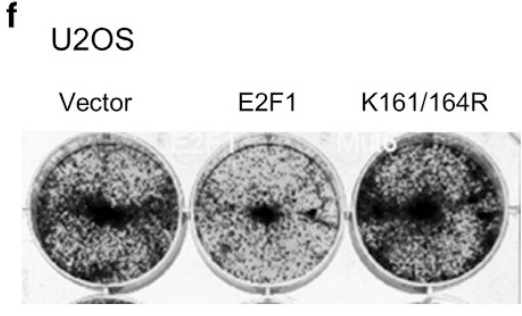

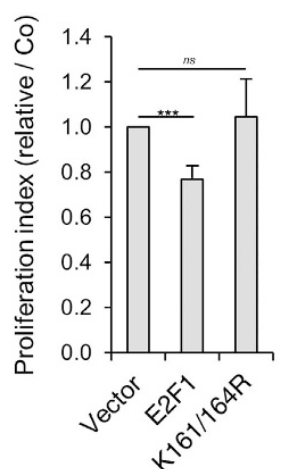

Figure 3 Cluster lysine 161 and 164 are important for E2F1 activity. (a and b) Gene luciferase experiments performed in Hela cells transfected with CCNE promoter-Firefly luciferase reporter plasmid and pCMV-3HA-E2F1 wt or E2F1 mutants constructs in which indicated K have been mutated into R (a) or (b) K0 (= mutation of every K into R), K89 only ( = mutation of every K into R except K89), K89, 137 ( = mutation of every K into R except K89 and K137) or K89, 137, 161, 164 (= mutation of every K into R except K89, K137, K161, K164). Luciferase activity was normalized to $\beta$-galactosidase activity. Results are expressed as $\%$ of activity measured with E2F1 wt. Mean \pm S.D. of at least three independent experiments. Statistical analysis performed using Student's $t$-test. ${ }^{* *} P<0.001,{ }^{* *} 0.001<P<0.01,{ }^{*} 0.01<P<0.1$, NS $P>0.1$. The expression of the constructs was checked by a western blot analysis (upper panel). $\beta$-Actin was used as loading control. (c-f) U2OS cells were transfected for $48 \mathrm{~h}$ with empty pCMV-3HA vector (V), pCMV-HA-E2F1 or pCM-3HA-E2F1 K161/164R mutant. (c) Western blot analysis of E2F1, $48 \mathrm{~h}$ after transfection. $\beta$-Actin was used as loading control. (d) Relative expression of ccne, tp73 and apaf1 mRNA as measured by RT-qPCR $48 \mathrm{~h}$ after transfection. Results were normalized to hprt mRNA and expressed relative to cells transfected with empty vector. Mean \pm S.D. of three independent experiments. (e) Chromatin IP experiments were performed using an anti-E2F1 or an irrelevant antibody (lgG). The levels of E2F1associated genomic DNA region encompassing E2F1 binding site of Cyclin $E(C C N E), T P 73$ or APAF1 promoter were quantified by qPCR. Results were normalized to input and the recruitment with irrelevant antibody, and expressed as relative recruitment compared with cells transfected with empty vector. (f) Proliferation of U2OS cells transfected for $48 \mathrm{H}$ with empty vector, E2F1 or E2F1 K161/164R mutant, as analyzed by crystal violet staining. Mean \pm S.D. of three independent experiments. Statistical analysis performed using Student's t-test. ${ }^{* *} P<0.001,{ }^{* *} 0.001<P<0.01,{ }^{*} 0.01<P<0.1$, NS $P>0.1$ 


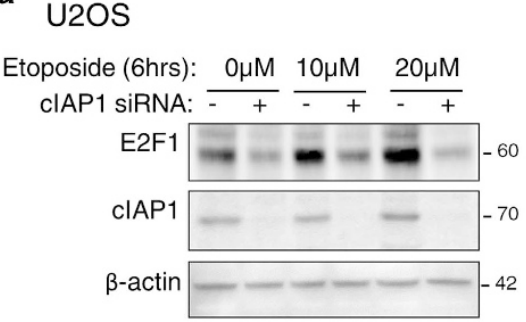

b

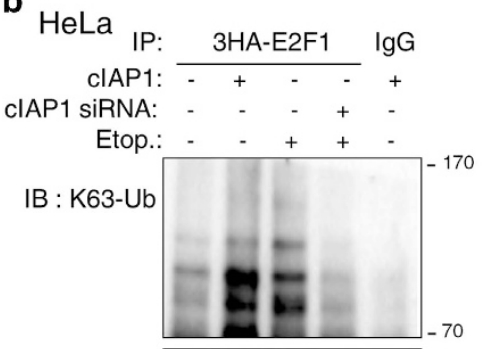

IB: E2F1 $\longrightarrow-70$ (3HA-E2F1)

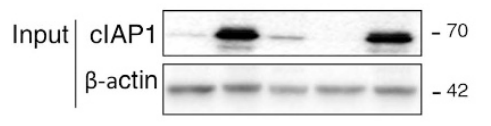

c
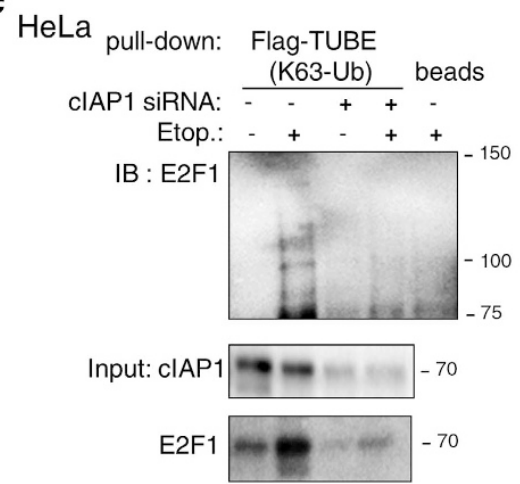

d

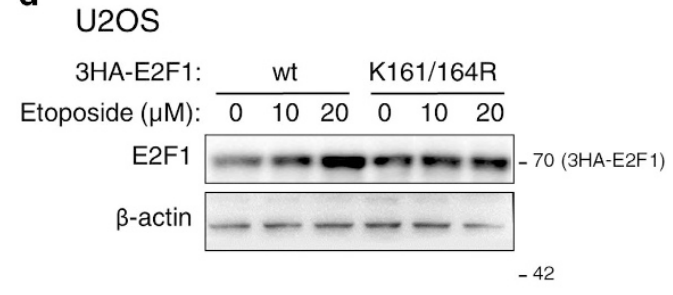

e

HeLa

IP:

$\mathrm{HA}$

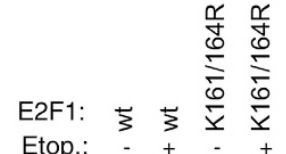

IB : K63-Ub

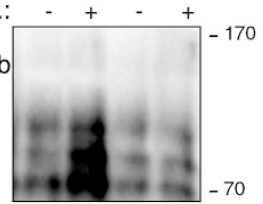

IB: E2F1 $=-70$ (3HA-E2F1)

f U2OS

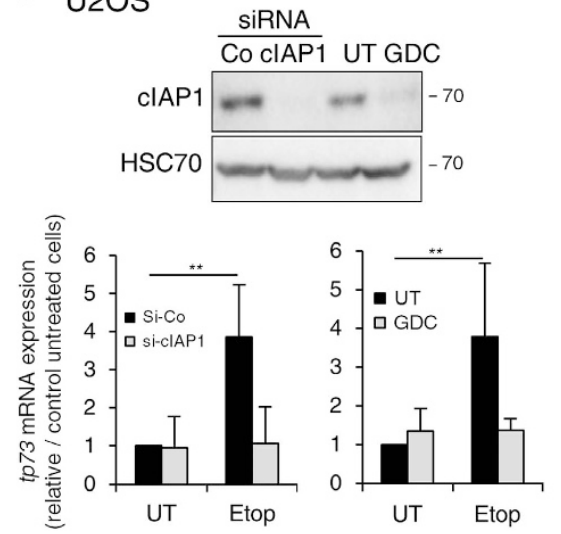

Figure 4 CIAP1 is required for DNA damage-induced stabilization of E2F1. (a) Western blot analysis of E2F1 and clAP1 in U2OS cells transfected with control or cIAP1siRNA and treated with indicated concentration of etoposide for $6 \mathrm{~h}$. $\beta$-Actin was used as loading control. (b) Ubiquitination profile of E2F1 in HeLa cells transfected with clAP1 siRNA or cIAP1-encoding construct and with 3HA-E2F1 and His-tagged ubiquitin wt. When indicated, cells were treated for $6 \mathrm{~h}$ with $10 \mu \mathrm{M}$ etoposide. E2F1 was IP using anti-HA antibody and ubiquitin revealed using K63-specific ubiquitin chain antibody (K63-Ub). The expression of the transgenes and the efficiency of siRNA were checked by a western blot analysis (input). $\beta$-Actin was used as loading control. (c) Ubiquitination assay performed in HeLa cells transfected with control or cIAP1 siRNA and with 3HA-E2F1 and His-tagged ubiquitin wt. When indicated, cells were treated for $6 \mathrm{~h}$ with $10 \mu \mathrm{M}$ etoposide. Ubiquitinated proteins were pulled-down by using K63 specific TUBEs and ubiquitinated E2F1 is revealed by using E2F1 antibody. (d) Western blot analysis of E2F1 in U2OS cells transfected with 3HA-E2F1 or 3HA-E2F1-K161/164R mutant and treated with indicated concentration of etoposide for $6 \mathrm{~h}$. $\beta$-Actin was used as loading control. (e) Ubiquitination of E2F1 in HeLa cells transfected with pCMV-3HA-E2F1 or pCMV-3HA-E2F1 $\mathrm{K} 161 / 164 \mathrm{R}$, and with His-tagged ubiquitin wt, and then treated for $6 \mathrm{~h}$ with $10 \mu \mathrm{M}$ etoposide. E2F1 was IP using anti-HA antibody and ubiquitin revealed using K63-specific ubiquitin chain antibody (K63-Ub). The level of expression of E2F1 constructs has been adjusted in order to get equivalent ubiquitin level in both untreated samples. (f) U2OS cells were transfected with cIAP1 siRNA or treated with $17 \mathrm{nM} \mathrm{GDC}-0152$ for $1 \mathrm{~h}$, and then treated with $10 \mu \mathrm{M}$ etoposide for $48 \mathrm{~h}$. tp73 mRNA expression was measured by RT-qPCR. UT: untreated cells. Results were normalized to hprt mRNA and expressed relatively to control untreated cells. Mean \pm S.D. of three independent experiments

induce a very quick degradation of $\operatorname{clAP} 1,{ }^{18}$ prevented etoposide from inducing the expression of the E2F1 target, DNA damage-responsive gene tp73 (Figure 4f).

The importance of arginine methylation in clAP1mediated accumulation of poly-ubiquitinated E2F1. The DNA damage-mediated E2F1 protein stability and activity is regulated by the arginine methyl-transferase PRMT-1. ${ }^{19}$ Interestingly, inhibition of PRMT-1 with its specific inhibitor
AMI-1 impaired the clAP1-mediated E2F1 upregulation (Figure 5a) and poly-ubiquitination (Figure 5b), as well as E2F1 transcriptional activity, in a dose-dependent manner (Figure 5c). Albeit to lower extend, AMI-1 also decreased etoposide-induced ubiquitination of E2F1 (Figure 5b). Accordingly, silencing of PRMT-1 partially prevented clAP1mediated E2F1 poly-ubiquitination (Figure 5d). Silencing of PRMT-5, another arginine methyl-transferase that promotes arginine methylation in cycling cells, ${ }^{20}$ also inhibited this 


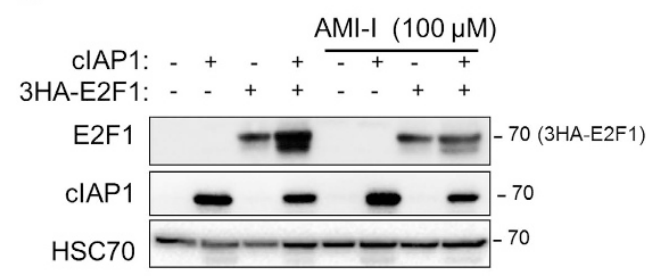

C

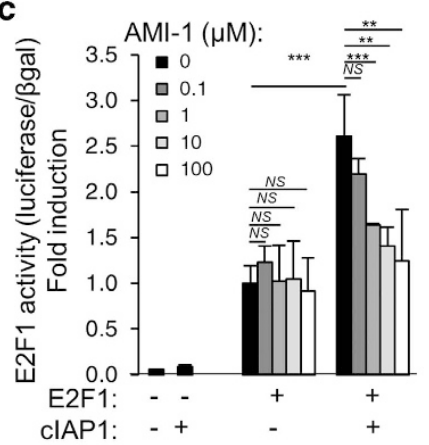

b

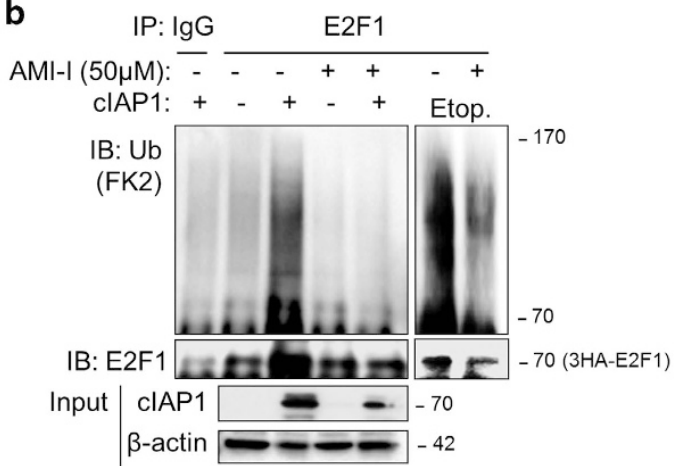

d
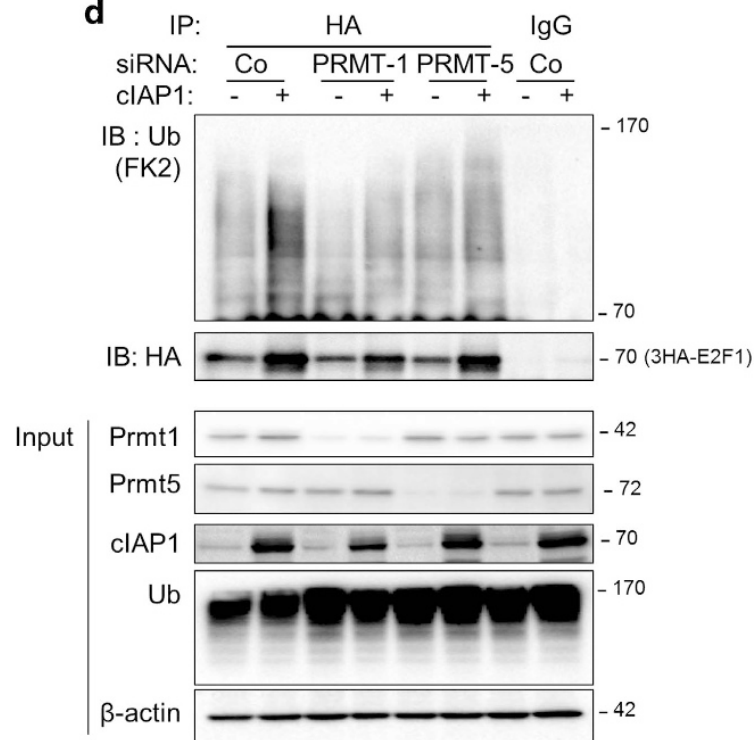

Figure 5 PRMT-mediated arginine methylation of E2F1 is required for regulation of E2F1 by clAP1. (a) Western blot analysis of E2F1 and clAP1 in HeLa cells transfected with 3HA-E2F1 and clAP1-encoding constructs, and treated for $24 \mathrm{~h}$ with $100 \mu \mathrm{M}$ PRMT inhibitor AMl-1. HSC70 was used as loading control. (b) Ubiquitination of E2F1 in HeLa

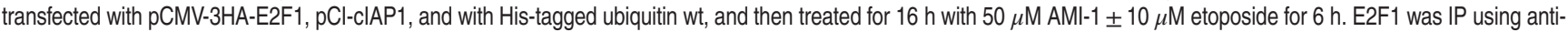
E2F1 antibody and ubiquitin revealed using pan anti-ubiquitin antibody (FK2). The expression of the transgene is checked by western blot. $\beta$-Actin was used as loading control. (c) E2F1 transcriptional activity as measured by using a gene reporter luciferase assay performed in Hela cells transfected as in a in the presence of CCNE promoter-Firefly luciferase reporter plasmid and treated with increasing concentration of AMl-1 for $24 \mathrm{~h}$. Results are expressed as average \pm S.D. from at least three experiments. Statistical analysis performed using Student's $t$-test. ${ }^{* \star}{ }^{*} P<0.001,{ }^{*} 0.001<P<0.01$, NS $P>0.1$. (d) Ubiquitination profile of E2F1 in HeLa transfected with Control (Co), PRMT-1 or PRMT-5-siRNA and pCMV-3HA-E2F1, pCl-cIAP1, and with His-tagged ubiquitin wt. E2F1 was IP using anti-HA antibody and ubiquitin revealed using pan anti-ubiquitin antibody (FK2). The expression of the transgene and the efficiency of siRNA were checked by western blot analysis. $\beta$-Actin was used as loading control

event (Figure 5d) suggesting that clAP1-mediated stabilization is likely to occur during the cell cycle.

\section{cIAP1-dependent K63 ubiquitination of E2F1 is cell cycle} regulated. The expression and the activity of E2F1 peak in early $S$ phase of cell cycle, and at the end of the $S$ phase, the cyclin A/cdk2 complex binds to and phosphorylates E2F1, leading to its inactivation and its subsequent degradation. We next assessed the role of CIAP1 and poly-ubiquitination of $\mathrm{E} 2 \mathrm{~F} 1$ in this regulatory process. A downregulation of cyclin A, which blocks cell cycle progression in late S-G2 (Supplementary Figure $3 \mathrm{~A}$ ) and freezes E2F1 in its $S$ phase, induced an increase in the expression of active E2F1 in both U2OS and HeLa cells (Figures 6a-c) and revealed a K63 ubiquitination of the transcription factor (Figure 6b). These two events were completely abolished by clAP1 depletion using siRNAs (Figures 6a and b). Consistent with the prominent role played by the lysines 161 and 164, the K161/164R E2F1 mutant resisted to cyclin A siRNA-mediated E2F1 accumulation (Figure $6 \mathrm{c}$ ) and $\mathrm{K} 63$ ubiquination (Figure $6 \mathrm{~d}$ ).

In the G1 phase, E2F1 is maintained in an inactive state because of its binding to $R b$ and phosphorylation of $R b$ is sufficient to release E2F1, allowing its activation and G1/S phase transition. Overexpression of $\mathrm{Rb}$ in HeLa cells, which are $\mathrm{Rb}$ deficient, did not affect the ubiquitination status of E2F1 (Supplementary Figure $3 \mathrm{~B}$ ) and downregulation of $\mathrm{Rb}$ in U2OS cells, which express wild-type Rb, slightly decreased E2F1 ubiquitination (Supplementary Figure $3 \mathrm{C}$ ), which is consistent with a decreased $S$ phase (Supplementary Figure 3A). Interestingly, overexpression of clAP1 in U2OS cells increased the phosphorylation of Rb (Figure 6e). Moreover, an in vitro competition experiment indicated that CIAP1 is able to compete with Rb for E2F1 binding (Supplementary Figure 3D), suggesting that ClAP1 may be likely to compete 
a

a u2OS

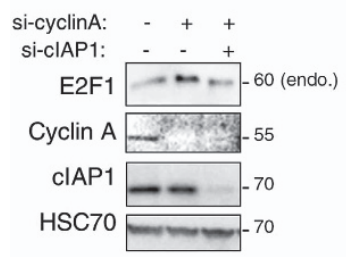

d HeLa

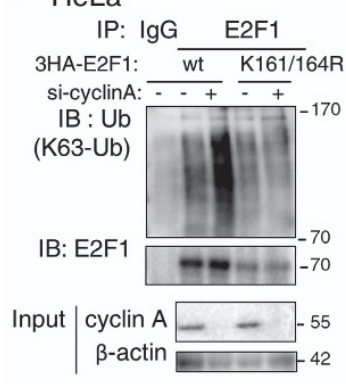

b

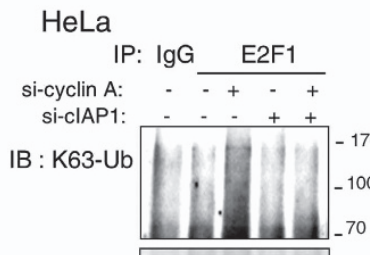

IB: E2F1 thet - 70 (3HA-E2F1)

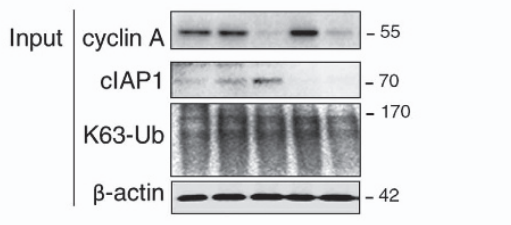

C HeLa

3HA-E2F1: wt K161/164R

si-cyclinA: $\frac{w t}{-+} \frac{K 161 / 104 R}{-}+$

E2F1 - - 70 (3HA-E2F1)

Cyclin A --55

$\beta$-actin $=-\infty-42$

e U2OS

$2 O S$ Co CIAP1

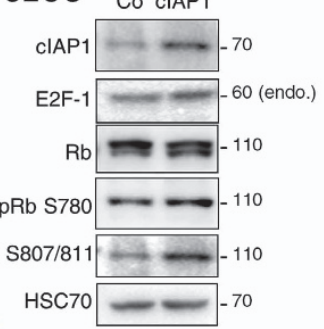

f

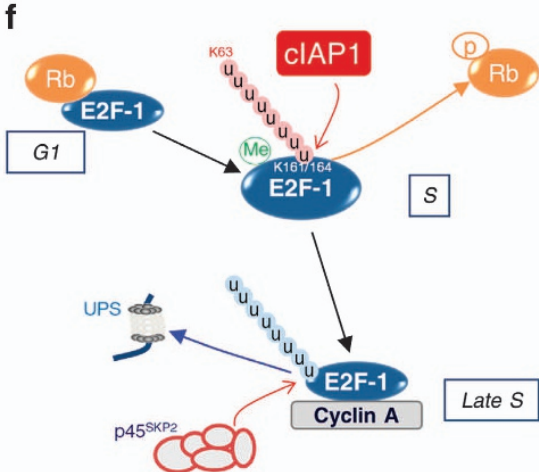

Figure 6 K63 ubiquitination of E2F1 is cell cycle regulated. (a) Western blot analysis of E2F1, cyclin A and clAP1 in U2OS cells transfected with cyclin A siRNA \pm clAP1 siRNA. HSC70 was used as loading control. (b) Ubiquitination profile of E2F1 in HeLa cells co-transfected with cyclin A siRNA \pm clAP1 siRNA, then $24 \mathrm{~h}$ later with pCMV-3HAE2F1 and His-tagged ubiquitin wt. E2F1 was IP using anti-E2F1 antibody and ubiquitin revealed using K63-specific ubiquitin chain antibody (K63-Ub). The expression of the transgenes was checked by a western blot analysis. $\beta$-Actin was used as loading control. (c) Western blot analysis of the expression of 3HA-E2F1 wt or K161/164 R mutant and cyclin A in HeLa cells transfected with cyclin A siRNA. $\beta$-Actin was used as loading control. (d) Ubiquitination profile of E2F1 in HeLa cells co-transfected with cyclin A siRNA, then $24 \mathrm{~h}$ later with pCMV-3HA-E2F1 or the K161/164 R mutant and His-tagged K63-only ubiquitin. E2F1 was IP using anti-E2F1 antibody and ubiquitin revealed using K63-specific ubiquitin chain antibody (K63-Ub). The expression of the transgenes was checked by a western blot analysis. $\beta$-Actin was used as loading control. The level of expression of E2F1 constructs has been adjusted in order to get equivalent ubiquitin level in both samples transfected with control siRNA. (e) Western blot analysis of clAP1 E2F1, cyclin E, Rb, phospho (S780, S807/811) Rb in U2OS cells transfected with clAP1-encoding construct. HSC70 was used as loading control. (f) Schematic representation of the regulation of E2F1. In the G1 phase of cell cycle, E2F1 is complexed to Rb. In the S phase, E2F1 is first methylated on arginine residue par PRMT, then K63-ubiquitinated on K161/164 in a CIAP1-dependent manner, leading to stabilization and activation of the protein. In the late S, cyclin A inhibits K63 ubiquitination of E2F1, binds to and phosphorylates E2F1 and promotes UPS-mediated degradation

with $\mathrm{Rb}$ for E2F1 binding during the cell cycle and thus to promote E2F1 activation (Figure 6f).

\section{Discussion}

The present report highlights a novel level of regulation of the stability and activity of E2F1 protein by a non-degradative ubiquitination process. The turnover of E2F1 is central to the regulation of its transcriptional activity. E2F1 expression peaks at the end of $\mathrm{G} 1$ phase of the cell cycle to promote the activation of genes required for the G1-S phase transition and the initiation of DNA synthesis. The expression of E2F1 also considerably rises in response to DNA damage, contributing to the apoptotic response to such damage. Genotoxic stressinduced stabilization of E2F1 was found to involve multiple interconnected post-translational modifications, that is, double-strand DNA breaks promote E2F1 phosphorylation on serine residues $31^{21}$ and $364,{ }^{22}$ its acetylation on lysine cluster 117, 120 and 125,,$^{23,24}$ its methylation at Arginine
$109,{ }^{19}$ its demethylation on lysine $185^{25}$ and arginine $111 / 113$ cluster $^{19}$ and its deNEDDylation. ${ }^{26}$ DNA damage was also found to promote E2F1 ubiquitination, ${ }^{27}$ but, so far, the E3-ubiquitin ligase involved remains unknown. Here, we demonstrate an accumulation of E2F1 K63-ubiquitinated on the lysines 161/164 cluster in cells treated with etoposide, as well as in those locked in the S phase, which is likely to involve the E3-ubiquitin ligase clAP1.

Although others IAPs including clAP2 and XIAP also behave as E3-ubiquitin ligases, only clAP1 stimulates E2F1 activity. ${ }^{12}$ clAP1, as a dimer, ${ }^{28,29}$ is able to promote the conjugation of $\mathrm{K} 11, \mathrm{~K} 48$ and $\mathrm{K} 63$ ubiquitin chains onto protein substrates. clAP1-mediated K11 and K63 ubiquitination was found to be involved in several ubiquitin-dependent cell signaling events, ${ }^{11}$ for example, in the regulation of NF- $\kappa \mathrm{B}$ activating and TNFR signaling pathways. More specifically, cIAP1-driven K11 and K63 ubiquitination of the kinase RIP1 is known to favors the assembly of a cell signaling protein platform that leads to NF- $\kappa \mathrm{B}$ activation, whereas preventing 
the formation of a RIP1-containing complex that induces cell death. ${ }^{30-33}$ A role of clAP1 in transcription regulation has also been described, for example, clAP1 promotes K48-linked ubiquitin chain conjugation and degradation of the myc antagonist Max-dimerization protein-1 (Mad-1), ${ }^{34}$ as well as the transcription factor CHOP (C/EBP homolog protein). ${ }^{35}$ Similar functions by other IAPs include the ability of XIAP to favor Wnt-dependent transcription program through mediating the mono-ubiquitination of Groucho (Gro)/TLE transcription co-repressor ${ }^{36}$ and the K63 ubiquitination of interferonregulatory factor 1 by clAP2. ${ }^{37}$

clAP1 is able to directly induce the ubiquitination of E2F1 and to promote the accumulation of E2F1 modified with K63-linkage ubiquitin chains on lysine residues 161 and 164. Additional experiments will be required to demonstrate that cIAP1 is indeed the E3-ubiquitine ligase responsible for the K63 ubiquitination of E2F1 in $\mathrm{S}$ phase of cell cycle or in response to etoposide. However, as E2F1 accumulation in such situations also involves ubiquitination of lysine residues 161 and 164, clAP1 is likely to be one of the E3-ligases responsible for these modifications. A K63 ubiquitination of E2F1 has recently been observed but the consequences were not clearly defined. ${ }^{9,10}$ K63-linked ubiquitin chains are the second most abundant ubiquitin signal in mammalian cells. $\mathrm{K} 63$ ubiquitination of proteins is involved in DNA repair, kinase activation, membrane protein internalization, intracellular vesicular trafficking and signaling protein complex assembly. ${ }^{5}$ Our data identified a function for K63 ubiquitination in cell cycle regulation through E2F1 protein stabilization. K63poly-ubiquitination may counteract the binding of other protein modifiers involved in protein degradation or favor the recruitment of protein chaperones. Accordingly, the mutation of lysine residues 161 and 164 was shown to strongly increase E2F1 NEDDylation. ${ }^{38}$ Stabilization of E2F1 requires PRMTmediated arginine methylation. Whereas PRMT-1 is important for E2F1 stabilization upon DNA damage, ${ }^{19} \mathrm{PRMT}-5$ promotes arginine methylation in cycling cells. ${ }^{20}$ Here, we show that a downregulation or an inhibition of either PRMT-1 or PRMT-5 decreases the clAP1-mediated E2F1 regulation, demonstrating the importance of a prior arginine methylation step.

Two main checkpoints control E2F1 activity during the S phase. The first one involves $\mathrm{Rb}$ that associates with and represses E2F1 before $S$ phase entry. Rb overexpression does not affect $\mathrm{K} 63$ ubiquitination of E2F1. Our results suggest that clAP1 is able to compete with Rb for E2F1 binding and that clAP1 overexpression is associated with an increases in $\mathrm{Rb}$ phosphorylation, indicating that clAP1 is likely to favor RbE2F1 dissociation to promote E2F1 activation and S phase initiation (Figure 6f). The second checkpoint is at the end of the $S$ phase, when cyclin A:Cdk2 complex binds to and phosphorylates E2F1, leading to the protein inactivation and subsequent degradation by skp2-dependent UPS. We identified an additional level of regulation of this checkpoint by demonstrating that cyclin A affects the K63 ubiquitination of E2F1 (Figure 6f).

The cluster of lysine 161 and 164 residues has been shown to be a target of E2F1 ubiquitination. In addition to completely abolishing the $S$ phase-dependent and the DNA damageinduced accumulation of E2F1, the mutation of K161/K164 cluster also radically impairs E2F1 transcriptional activity, that is, its capacity to bind CCNE, TP73 and APAF1 gene promoters. Consistent with these observations, downregulation of clAP1 was found to block the etoposide-induced expression of the E2F1 transcriptional target TP73. These results are consistent with our previous work demonstrating that silencing of clAP1 inhibits E2F1-dependent expression of CCNE and CCNA genes in cycling cells through inhibition of E2F1 recruitment on the corresponding gene promoters. ${ }^{12} \mathrm{We}$ also showed that clAP1, which interacts with E2F1 in all phases of the cell cycles, is recruited, along with E2F1, onto E2F-binding sites of $C C N E$ and $C C N A$ promoters during the $S$ phase, when E2F1 activity is at its maximum. ${ }^{12}$ Our current results suggest that E2F1 ubiquitination is key in inducing E2F1 interaction with these target gene promoters, possibly through recruitment of transcriptional co-regulators containing ubiquitin binding modules ${ }^{39}$ to form transcriptional complexes. The deubiquitinases (DUB) $\mathrm{POH} 1$ and $\mathrm{UCH} 37$, both described as a K63-DUB, were found to regulate E2F1 stability or activity, although with opposite effects. ${ }^{9,10} \mathrm{POH} 1-$ mediated deubiquitination appeared to lead to E2F1 stabilization, ${ }^{10}$ whereas $\mathrm{UCH} 37$-mediated deubiquitination enhanced E2F1 transcriptional activity. ${ }^{9}$ The role of each of these two DUB in removing DNA damage and $S$ phaseassociated $\mathrm{K} 63$ ubiquitin chains from E2F1 merits further investigations. Mutation of E2F1 K161/164 does not completely abolish E2F1 K63 ubiquitination profile (Figure 2d) meaning that other lysine residues may be conjugated with K63 ubiquitin chains.

In summary, we demonstrated that CIAP1 as an E3-ubiquitin ligase is able to promote the accumulation of E2F1 K63ubiquinated on the clustered lysine residues 161 and 164 . Such clAP1-dependent E2F1 modifications was observed both in the $S$ phase of the cell cycle and in response to DNA damage, two situations in which E2F1 transcriptional activity is optimal, suggesting a general mechanism of E2F1 activity regulation. Our findings suggest that IAP-targeting molecules could be tested for their ability to overcome E2F1 activity in tumor cells, for example, in an Rb-compromised setting.

\section{Materials and Methods}

Cell culture and treatment. Human cervical carcinoma cell line (HeLa), human osteosarcoma epithelial cell line (U20S) and mouse embryonic immortalized (SV40) fibroblasts (MEF) ClAP1 $1^{-1-/ C I A P 2} 2^{-1-}$ (J Silke, Melbourne, Australia), were grown in Dulbecco's modified Eagle's medium (Dominique Dutscher, Brumath, France) or RPMI 1640 (Roswell Park Memorial Institute, Dominique Dutscher) containing $10 \%$ fetal bovine serum (Dominique Dutscher) at $37^{\circ} \mathrm{C}$ in a $5 \% \mathrm{CO}_{2}$ atmosphere and $95 \%$ humidity. Cells were treated with 10 or $20 \mu \mathrm{M}$ etoposide (Sigma-Aldrich, Lyon, France) for $6 \mathrm{~h}, 100 \mu \mathrm{g} / \mathrm{ml}$ cycloheximide (Sigma-Aldrich), 10-35 $\mu$ M PYR-41 (Calbiochem, Merck Millipore, Saint-Quentin en Yvelines, France) for $24 \mathrm{~h}, 0.1-100 \mu \mathrm{M}$ AMI-I (Enzo Life Sciences, Villeurbanne, France) for $24 \mathrm{~h}$, or $1 \mu \mathrm{M}$ GDC-0152 (Selleckmed, Euromedex, Souffelweyersheim, France). MG132 (Sigma-Aldrich,) was used at $1 \mu \mathrm{M}$ or $5 \mu \mathrm{M}$ overnight.

Plasmid constructs and siRNA transfections: Plasmids used were $\mathrm{pCl}$ empty vector, pCl-clAP1, pCl-clAP1-H588A, pCMV- $\beta$ Gal, pCMV-HA-DP1, pGL3 human CCNE promoter; ${ }^{12}$ pcDNA3.1-6His-Ub wt and 6His-Ub-K63-only mutants in which all $\mathrm{K}$ have been mutated into $\mathrm{R}$ except the K63; pCMV-HA-E2F1 wt and pCMVHA-E2F1 K117/120/125R, ${ }^{17}$ pCMV-3HA-E2F1 and pCMV-3HA-E2F1-K185R; ${ }^{25}$ pCMV-Flag-E2F1 wt and mutants that contain no lysine (K0) or only K89 (K89 only), K89 and K137 (K89, 137), K89, K137, K161 and K164 (K89, 137, 161, 164) or in which K117/120/125) (cluster 1), K182/183/185 (cluster 2) and/or K226/287/ 289/310 (cluster 3 ) have been mutated into R. $^{26}$ The pCl-clAP1-F616A mutant was 
obtained by site-directed mutagenesis from pCl-clAP1 with GENEART Site-Directed Mutagenesis system (Invitrogen, ThermoFisher Scientific, Villebon-sur-Yvette, France) by using the following primers: forward (5'-CAAGGGTACTGTTCGTACAGCTC TCTCATAATCGACCCG-3'), reverse (5'-CGGGTCGATTATGAGAGAGCTGTACGA ACAGTACCCTTG-3'). The pCMV-3HA-E2F1 mutants (K89R, K137R, K161/164R, K181/183R, K266R, K287/289R, K310R) were obtained by site-directed mutagenesis from pCMV-3HA-E2F1 (GenScript, Piscataway, NJ, USA). Plasmids constructs were transiently transfected using JetPEI (Polyplus transfection, Ozyme, Montigny-leBretonneux, France). Lipofectamine RNAimax reagent (ThermoFisher Scientific, Villebon-sur-Yvette, France) was used to transfect siRNAs. RNA oligonucleotides are designed and purchased from Qiagen (Qiagen France SAS, Courtaboeuf, France) for clAP1, Rb, cyclin A and control siRNA or Ambion (Thermo Fisher Scientific) for PRMT-1 and PRMT-5 SIRNA.

Gene reporter assay. The transactivation activity of E2F1 was analyzed as previously described. ${ }^{12}$

Cellular extracts and western blot analysis: Cells were lysed in RIPA buffer ( $\mathrm{NaCl} 150 \mathrm{mM}, \mathrm{NP} 401 \%$, deoxycholate sodium (DOC) 0.5\%, SDS 0.1\%, Tris pH $7.550 \mathrm{mM}$ and protease inhibitor cocktail). Proteins were separated on SDS-PAGE and electro-transferred onto polyvinylidene difluoride membranes (GE Healthcare, Dominique Dutscher, Brumath, France). Blots were probed with the following antibodies: a rabbit polyclonal anti-E2F1 (C20), a mouse monoclonal anti-HSC70 and a mouse monoclonal anti-PRMT-5 (A-11) from Santa Cruz Biotechnology (Santa Cruz Biotechnology, Clinisciences, Naterre, France), a goat polyclonal antiCIAP1 from R\&D Systems (Minneapolis, MN, USA) (AF8181); a rabbit polyclonal anti-PRMT-1 from Upstate, Upstate Biotechnology (Merck Millipore), a mouse anti$\beta$-actin (AL978) and a mouse anti- $\alpha$-tubulin (clone AA13) from Sigma-Aldrich (Lyon, France), a monoclonal FK2-HRP-conjugated anti-ubiquitin (mono and poly-ubiquitin chains), a monoclonal HRP-conjugated anti-K63 linkage-specific ubiquitin (HWA4C4) from Enzo Life Sciences, a mouse monoclonal anti-HA.11 (Biolegend, San Diego, CA, USA), a mouse anti-cyclin A (BD Biosciences Grenoble, France), a rabbit anti-Rb and a rabbit anti-phospho-Rb (Ser780 or Ser807/811) (Cell Signaling Technology, Ozyme, Saint-Quentin en Yvelines, France). Detection was performed using peroxidase-conjugated secondary antibodies and chemiluminescence detection kit (ClarityTM western ECL substrate, Bio-Rad, Marnes-la-Coquette, France).

Ubiquitinylation assay. For the in vitro ubiquitination assay, GST-E2F1 fusion protein was produced in Escherichia coli by using the pGEX-E2F1 construct, immobilized on gluthatione sepharose beads (GE Healthcare) and incubated Sigma at $37^{\circ} \mathrm{C}$ for $1 \mathrm{~h}$ with recombinant CIAP1 (R\&D System), $100 \mathrm{nM}$ of recombinant human E1 ubiquitin-activating enzyme UBE1 (Boston Biochem, Cambridge, MA, USA), $1.5 \mu \mathrm{g}$ of recombinant human E2 enzyme Ubc H5a/UBE2D1 (Boston Biochem), $0.2 \mathrm{mM}$ of recombinant human ubiquitin (R\&D Systems) in a buffer containing $75 \mathrm{mM}$ Tris pH $8.2 \mathrm{mM}$ DTT, $5 \mathrm{mM}$ de $\mathrm{MgCl}_{2}, 4 \mathrm{mM}$ ATP. Gluthatione sepharose beads were then washed and proteins were eluted in Leammli buffer. E2F1 ubiquitination was revealed using anti-E2F1 antibody immunoblotting.

For the ubiquitination assays, HeLa cells were transfected with $5 \mu \mathrm{g}$ of each plasmid encoding ubiquitin wt or K63-only mutant, HA-E2F1 wt or mutants, CIAP1 wt or mutants. Cells were treated overnight with $1 \mu \mathrm{M}$ MG132 (Sigma) and with the nonselective DUB inhibitors PR619 10 (LifeSensors, Tebu-bio SAS, France) before cell lysis. Ubiquitinated proteins were pulled-down following the manufacturer instructions by using Flag-conjugated anti-K63 TUBEs, Agarose-TUBEs or GST-TUBEs (LifeSensors) and revealed by using anti-E2F1 antibody. For the analysis of ubiquitination profile of E2F1, 3HA-E2F1 was immunoprecipitated (IP) with anti-E2F1 or anti-HA antibody and revealed by using monoclonal FK2-HRP-conjugated antiubiquitin (mono and poly-ubiquitin chains) or a monoclonal HRP-conjugated anti-K63linkage specific ubiquitin (HWA4C4) (Enzo Life Sciences).

Immunoprecipitation: Cells ( $10^{6}$ per conditions) were transfected with plasmids encoding E2F1 wt or K161/164 R mutant, cIAP1 wt or DP1. Forty-eight hours after transfection, cells were washed with PBS and lysed in IP Lysis buffer $(150 \mathrm{mM}$ de $\mathrm{NaCl}, 50 \mathrm{mM}$ Tris $\mathrm{HCl}$ pH 7,4, $20 \mathrm{mM}$ EDTA, 0,5\% NP40, $1 \mathrm{mM}$ DTT, $5 \mathrm{mM}$ $\mathrm{N}$-ethylmaleimide (Sigma-Aldrich) and protease inhibitors) for $30 \mathrm{~min}$ at $4{ }^{\circ} \mathrm{C}$. Lysate supernatants were precleaned by using $20 \mu \mathrm{l}$ of Protein $\mathrm{A} / \mathrm{G}+$ Agarose beads (Sigma) and incubated (overnight, $4^{\circ} \mathrm{C}$ ) with rabbit polyclonal anti-E2F1 (C20) antibody (Santa Cruz Biotechnology), mouse lgG1 purified anti-HA.11 (Biolegend), goat polyclonal anti-clAP1 (R\&D Systems) or lgg anti-mouse or anti-rabbit antibody. Beads were then washed in IP lysis buffer and denaturated in Laemmli buffer before immunoblot analysis.

GST-pull down analysis: GST-pull down analysis was performed as previously described. ${ }^{40}$

RNA Purification, reverse transcription, real-time PCR ( $q P C R)$ : Total RNAs were isolated using TRIzol Reagent (Invitrogen, ThermoFisher Scientific). RNA quality was ascertained using a nanospectrophotometer (Nanodrop 2000, Thermo Scientific, Waltham, MA, USA). Total RNA $(1 \mu \mathrm{g})$ was reverse- transcribed using iScript cDNA synthesis kit (Bio-Rad) according to manufacturer's instructions. Quantitative real-time PCR was performed with 7500 Fast thermocycler or StepOne plus system (Applied Biosystem, ThermoFisher Scientific) using SYBR Green detection protocol and the following primers: TP73 promoter forward: $5^{\prime}$-TGAGCCA TGAAGATGTGCGAG-3', reverse: 5'-GCTGCTTATGGTCTGATGCTTATG-3'; APAF1 promoter forward: 5'-GGAGACCCAGGACGACAA-3'; reverse: $5^{\prime}$-CAGTGA AGCAACGAGGATGC-3'; CCNE promoter forward: $55^{\prime}$-CCATCGGCCATCTTCC TGGCTC-3', reverse: $5^{\prime}$-TCAGGCCGCGGGCCCAGTA-3'; CCNE forward: 5'-GCC AGCCTTGGGACAATAATG-3', reverse: 5'-CTTGCACGTTGAGTTTGGGT-3'; P73 forward: 5'-GACGAGGACACGTACTACCTT-3', reverse: 5'-CTGCCGATAGGAGTCCA CCA-3'; APAF1 forward: 5'-TCACTGCAGATTTTCACCAGA-3', reverse: 5'-CCTCTC ATTTGCTGATGTCG-3'; HPRT forward: 5'-GGACAGGACTGAACGTCTTGC-3', reverse: 5'-CTTGAGCACACAGAGGGCTACA-3'.

Chromatin IP: Hela cells $\left(1.5 \times 10^{6}\right.$ per conditions) were transfected with $5 \mu \mathrm{g}$ of pCMV-3HA-E2F1 wt or K161/164 R mutant encoding vectors and $10 \mu \mathrm{g}$ of $\mathrm{PCl}$ control vector, then protein/DNA complexes were cross-linked with formaldehyde $1 \%$. Chromatid was isolated and digested with micrococcal nuclease. Ten percent of lysate containing the digested chromatid was conserved for input. The remaining lysate is diluted and IP using $5 \mu \mathrm{g}$ of rabbit anti-E2F1 (C20) or rabbit control Igg (Santa Cruz Biotechnology) overnight. Elution and DNA recovery were performed according to recommendation of PIERCE Agarose Chip Kit (Thermo Scientific). Specific chromatin associated with interest protein was analyzed by $\mathrm{qPCR}$ as described above.

Cell proliferation: U2OS cells were transfected with pCMV-3HA-E2F1 wt or K161/164 R mutant encoding vectors. Twenty-four hours later, transfected 20000 U2OS cells were implanted in six-well plates for 6 days. Six days later, cells were fixed by ethanol $(100 \%)$ and stained with crystal violet. Crystal violet staining was eluted with acetic acid (33\%) and the optical density at $620 \mathrm{~nm}$ was measured with a plate reader (UVM 340, Biochrom, Cambourne, UK).

Cell cycle analysis: U2OS cells $\left(0.65 \times 10^{5}\right.$ per conditions) were transfected with $20 \mu \mathrm{M}$ of control or cyclin A-siRNA, or $50 \mu \mathrm{M} \mathrm{Rb}$-siRNA. Cell cycle is evaluated by using the BrdU Flow kit (BD Bioscience) $48 \mathrm{~h}$ after transfection according to the manufacturer instruction and cell cycle repartition was assessed by BD FACS Canto II flow cytometer using Flowing Software 2 (Call imaging Core, Turku Centre for Biotechnology, Turku, Finland).

Statistical analysis: Student's t-test or the Mann-Whitney Wilcoxon test was used for statistical analysis.

\section{Conflict of Interest}

The authors declare no conflict of interest.

Acknowledgements. We thank Dr Plenchette for His-ub mutant constructs, $\mathrm{Dr}$ Gotoh, Dr Giacca and Dr Talianidis for E2F1 mutant constructs, Dr LeRomancer for PRMT antibodies. We thank Catherine Paul for enriching discussions and proof reading of the manuscript. This work was supported by grants from the 'Comités de Côte d'Or et de l'Yonne' of the 'Ligue Contre le Cancer' (LD), the 'Association pour la Recherche sur le Cancer' (ARC to LD), the European Union and the 'Conseil Régional de Bourgogne', a French Government grant managed by the French National Research Agency under the program 'Investissements d'Avenir' with reference ANR-11-LABX-0021, and fellowships from the 'Ministère de l'Enseignement Supérieur et de la Recherche' of France (to JA, JB and JC), 'Ligue Nationale Contre le Cancer' (VG) and the "Fondation pour la Recherche Médicale (FRM) (to JB). 


\section{Author contributions}

$V G$ and $J A$ a performed most of the experiments. JB first identified the ubiquitination of E2F1 and initiated the work. BD, P-MB, ND, CK and JC performed additional western blot analysis and gene reporter assays. SG performed GST-pull down experiments. GM and DG performed analysis of E2F1 and its mutants, RB brought expertise in ChIP analysis. CG, OM and ES corrected the paper and LD conceived and supervised the project, analyzed the data and wrote the paper with input from all authors.

1. Laine A, Westermarck J. Molecular pathways: harnessing E2F1 regulation for prosenescence therapy in p53-defective cancer cells. Clin Cancer Res 2014; 20: 3644-3650.

2. Munro S, Carr SM, La Thangue NB. Diversity within the pRb pathway: is there a code of conduct? Oncogene 2012; 31: 4343-4352.

3. Bertoli C, Skotheim JM, de Bruin RA. Control of cell cycle transcription during G1 and S phases. Nat Rev Mol Cell Biol 2013; 14: 518-528.

4. Biswas AK, Johnson DG. Transcriptional and nontranscriptional functions of E2F1 in response to DNA damage. Cancer Res 2012; 72: 13-17.

5. Erpapazoglou Z, Walker 0 , Haguenauer-Tsapis R. Versatile roles of k63-linked ubiquitin chains in trafficking. Cells 2014; 3 : 1027-1088.

6. Marti A, Wirbelauer $C$, Scheffner M, Krek W. Interaction between ubiquitin-protein ligase SCFSKP2 and E2F-1 underlies the regulation of E2F-1 degradation. Nat Cell Biol 1999; 1: $14-19$

7. Peart MJ, Poyurovsky MV, Kass EM, Urist M, Verschuren EW, Summers MK et al. APC/C (Cdc20) targets E2F1 for degradation in prometaphase. Cell Cycle 2010; 9: 3956-3964.

8. Budhavarapu VN, White ED, Mahanic CS, Chen L, Lin FT, Lin WC. Regulation of E2F1 by APC/C Cdh1 via K11 linkage-specific ubiquitin chain formation. Cell Cycle 2012; 11: 2030-2038

9. Mahanic CS, Budhavarapu V, Graves JD, Li G, Lin WC. Regulation of E2 promoter binding factor 1 (E2F1) transcriptional activity through a deubiquitinating enzyme, UCH37. J Biol Chem 2015; 290: 26508-26522.

10. Wang B, Ma A, Zhang L, Jin WL, Qian $Y, X u$ G et al. POH1 deubiquitylates and stabilizes E2F1 to promote tumour formation. Nat Commun 2015; 6: 8704

11. Estornes $Y$, Bertrand MJ. IAPs, regulators of innate immunity and inflammation. Semin Cell Dev Biol 2014; 39: 106-114.

12. Cartier J, Berthelet J, Marivin A, Gemble S, Edmond V, Plenchette S et al. Cellular inhibitor of apoptosis protein-1 (clAP1) can regulate E2F1 transcription factor-mediated control of cyclin transcription. J Biol Chem 2011; 286: 26406-26417.

13. Che X, Yang D, Zong H, Wang J, Li X, Chen F et al. Nuclear clAP1 overexpression is a tumor stage- and grade-independent predictor of poor prognosis in human bladder cancer patients. Urol Oncol 2012; 30: 450-456.

14. Plenchette S, Cathelin S, Rebe C, Launay S, Ladoire S, Sordet O et al. Translocation of the inhibitor of apoptosis protein c-IAP1 from the nucleus to the Golgi in hematopoietic cells undergoing differentiation: a nuclear export signal-mediated event. Blood 2004; 104: 2035-2043

15. Tanimoto T, Tsuda $\mathrm{H}$, Imazeki N Ohno Y, Imoto I, Inazawa J et al. Nuclear expression of ClAP-1, an apoptosis inhibiting protein, predicts lymph node metastasis and poor patient prognosis in head and neck squamous cell carcinomas. Cancer Lett 2005; 224: 141-151.

16. Feltham R, Bettjeman B, Budhidarmo R, Mace PD, Shirley S, Condon SM et al. Smac mimetics activate the E3 ligase activity of clAP1 protein by promoting RING domain dimerization. J Biol Chem 2011; 286: 17015-17028.

17. Marzio G, Wagener $C$, Gutierrez Ml, Cartwright $P$, Helin $K$, Giacca M. E2F family members are differentially regulated by reversible acetylation. J Biol Chem 2000; 275: 10887-10892.

18. Flygare JA, Beresini M, Budha N, Chan H, Chan IT, Cheeti S et al. Discovery of a potent small-molecule antagonist of inhibitor of apoptosis (IAP) proteins and clinical candidate for the treatment of cancer (GDC-0152). J Med Chem 2012; 55: 4101-4113.

19. Zheng S, Moehlenbrink J, Lu YC, Zalmas LP, Sagum CA, Carr S et al. Arginine methylationdependent reader-writer interplay governs growth control by E2F-1. Mol Cell 2013; 52: 37-51.

20. Cho EC, Zheng S, Munro S, Liu G, Carr SM, Moehlenbrink J et al. Arginine methylation controls growth regulation by E2F-1. EMBO J 2012; 31: 1785-1797.

21. Lin WC, Lin FT, Nevins JR. Selective induction of E2F1 in response to DNA damage, mediated by ATM-dependent phosphorylation. Genes Dev 2001; 15: 1833-1844.
22. Stevens $C$, Smith L, La Thangue NB. Chk2 activates E2F-1 in response to DNA damage. Nat Cell Biol 2003; 5: 401-409.

23. Pediconi N, lanari $A$, Costanzo A, Belloni L, Gallo R, Cimino $L$ et al. Differential regulation of E2F1 apoptotic target genes in response to DNA damage. Nat Cell Biol 2003; 5: 552-558.

24. Ianari A, Gallo R, Palma M, Alesse E, Gulino A. Specific role for p300/CREB-binding proteinassociated factor activity in E2F1 stabilization in response to DNA damage. J Biol Chem 2004; 279: 30830-30835.

25. Kontaki $H$, Talianidis I. Lysine methylation regulates E2F1-induced cell death. Mol Cell 2010; 39: 152-160.

26. Aoki I, Higuchi M, Gotoh Y. NEDDylation controls the target specificity of E2F1 and apoptosis induction. Oncogene 2013; 32: 3954-3964.

27. Galbiati L, Mendoza-Maldonado R, Gutierrez MI, Giacca M. Regulation of E2F-1 after DNA damage by $\mathrm{p300-mediated} \mathrm{acetylation} \mathrm{and} \mathrm{ubiquitination.} \mathrm{Cell} \mathrm{Cycle} \mathrm{2005;} \mathrm{4:} \mathrm{930-939.}$

28. Nakatani Y, Kleffmann T, Linke K, Condon SM, Hinds MG, Day CL. Regulation of ubiquitin transfer by XIAP, a dimeric RING E3 ligase. Biochem J 2013; 450: 629-638.

29. Feltham R, Moulin M, Vince JE, Mace PD, Wong WW, Anderton $\mathrm{H}$ et al. Tumor necrosis factor (TNF) signaling, but not TWEAK (TNF-like weak inducer of apoptosis)-triggered clAP1 (cellular inhibitor of apoptosis protein 1) degradation, requires cIAP1 RING dimerization and E2 binding. J Biol Chem 2010; 285: 17525-17536.

30. Dynek JN, Goncharov T, Dueber EC, Fedorova AV, Izrael-Tomasevic A, Phu L et al. c-IAP1 and $\mathrm{UbcH} 5$ promote $\mathrm{K} 11$-linked polyubiquitination of RIP1 in TNF signalling. EMBO J 2010; 29: 4198-4209.

31. Varfolomeev E, Goncharov T, Fedorova AV, Dynek JN, Zobel K, Deshayes K et al. c-IAP1 and C-IAP2 are critical mediators of tumor necrosis factor alpha (TNFalpha)-induced NF-kappaB activation. J Biol Chem 2008; 283: 24295-24299.

32. Bertrand MJ, Milutinovic S, Dickson KM, Ho WC, Boudreault A, Durkin J et al. clAP1 and cIAP2 facilitate cancer cell survival by functioning as E3 ligases that promote RIP1 ubiquitination. Mol Cell 2008; 30: 689-700.

33. de Almagro MC, Goncharov T, Newton K, Vucic D. Cellular IAP proteins and LUBAC differentially regulate necrosome-associated RIP1 ubiquitination. Cell Death Dis 2015; 6 : e1800.

34. Xu L, Zhu J, Hu X, Zhu H, Kim HT, LaBaer J et al. c-IAP1 cooperates with Myc by acting as a ubiquitin ligase for Mad1. Mol Cell 2007; 28: 914-922.

35. Qi Y, Xia P. Cellular inhibitor of apoptosis protein-1 (clAP1) plays a critical role in beta-cell survival under endoplasmic reticulum stress: promoting ubiquitination and degradation of C/EBP homologous protein (CHOP). J Biol Chem 2012; 287: 32236-32245.

36. Hanson AJ, Wallace HA, Freeman TJ, Beauchamp RD, Lee LA, Lee E. XIAP monoubiquitylates Groucho/TLE to promote canonical Wnt signaling. Mol Cell 2012; 45 $619-628$

37. Harikumar KB, Yester JW, Surace MJ, Oyeniran C, Price MM, Huang WC et al. K63-linked polyubiquitination of transcription factor IRF1 is essential for IL-1-induced production of chemokines CXCL10 and CCL5. Nat Immunol 2014; 15: 231-238.

38. Loftus SJ, Liu G, Carr SM, Munro S, La Thangue NB. NEDDylation regulates E2F-1. dependent transcription. EMBO Rep 2012; 13: 811-818.

39. Rahighi S, Dikic I. Selectivity of the ubiquitin-binding modules. FEBS Lett 2012; 586 2705-2710.

40. Marivin A, Berthelet J, Cartier J, Paul C, Gemble S, Morizot A et al. clAP1 regulates TNF-mediated cdc42 activation and filopodia formation. Oncogene 2014; 33: 5534-5545.

(c) (i) Cell Death and Disease is an open-access journal published by Nature Publishing Group. This work is licensed under a Creative Commons Attribution 4.0 International License. The images or other third party material in this article are included in the article's Creative Commons license, unless indicated otherwise in the credit line; if the material is not included under the Creative Commons license, users will need to obtain permission from the license holder to reproduce the material. To view a copy of this license, visit http://creativecommons.org/licenses/by/4.0/

(C) The Author(s) 2017

Supplementary Information accompanies this paper on Cell Death and Disease website (http://www.nature.com/cddis) 